\title{
FACTORABLE BOUNDED OPERATORS AND SCHWARTZ SPACES
}

\author{
STEVEN F. BELLENOT
}

\begin{abstract}
A necessary condition for factoring continuous linear maps with domain $c_{0}$ or $l_{\infty}$ through a class of spaces which include the $l_{p}$ spaces (in fact, include the $\mathscr{L}_{p}$ spaces) for $2 \leqq p<\infty$ and a weaker result for $l_{1}$ are obtained. As an application, examples of Schwartz spaces are constructed and used to answer questions of Diestel, Morris and Saxon; in particular it is shown that there are Schwartz spaces which cannot be embedded in a product of $l_{p}$ spaces, $1<p<\infty$.
\end{abstract}

For $2 \leqq p<\infty$, we show the following: If $S$ is a continuous linear map from $c_{0}$ or $l_{\infty}$ into a normed space, then $S$ is factorable through a space satisfying Clarkson's "parallelogram" laws for $L_{p}$ spaces ([1] or see (1) below) only if a sequence, which is simply constructed from $S$, belongs to $l_{p}$. A similar result is obtained for diagonal maps on $l_{1}$. Examples are constructed to show that for no $p, 1<p<\infty$, does the variety $v\left(l_{p}\right)$ (defined below) contain the variety of all Schwartz spaces [5, p. 275]. In particular this shows that the theorem of Grothendieck [4] that any nuclear space can be topologically embedded in a product of $l_{p}$ spaces, $1 \leqq p \leqq \infty$, (and Saxon's generalization [9]) cannot be extended to Schwartz spaces. Furthermore, an example borrowed from Pietsch [8] shows that the variety of nuclear spaces is properly contained in $\bigcap v\left(l_{p}\right)$, $1 \leqq p \leqq \infty$. These examples answer or partially answer questions raised by Diestel, Morris and Saxon in [3] or by Diestel and Morris in [2].

By a map we shall mean a continuous linear function. To say a map $S: X \rightarrow Y$ can be factored through $Z$ means that there exist maps $T: X \rightarrow Z$ and $J: Z \rightarrow Y$ such that $S=J T$. Let $e_{n}$ be the sequence with one in the $n$th place and zero otherwise. A variety (see [2] or [3]) is a collection of locally convex topological vector spaces closed with respect to taking subspaces, quotients by closed subspaces, products and isomorphic images. If $B$ is a locally convex topological vector space, we let $\nu(B)$ be the

Received by the editors March 26, 1973 and, in revised form, April 30, 1973.

AMS (MOS) subject classifications (1970). Primary 46E30, 46A15; Secondary 47A99, 47B99.

Key words and phrases. Factorable maps, $l_{p}$-spaces, Schwartz spaces, nuclear spaces, varieties of topological vector spaces.

(c) American Mathematical Society 1974 
smallest variety containing $B$. For $2 \leqq p<\infty$, it is convenient to define a $Q_{p}$ space to be any normed space $X$ which has an equivalent norm $\|\cdot\|$ satisfying

$$
\forall x, y \in X, \quad\|x+y\|^{p}+\|x-y\|^{p} \geqq 2\left(\|x\|^{p}+\|y\|^{p}\right) .
$$

Clarkson, in [1, Theorem 2, p. 400], shows that the usual norms of $L_{p}$ and $l_{p}$ satisfy (1) for $2 \leqq p<\infty$. It is easy to show that the property of being a $Q_{p}$ space is preserved under taking subspaces, quotients by closed subspaces, and finite products. Thus any $\mathscr{L}_{p}$ space (see [6] or [7]) is a $Q_{p}$ space for $2 \leqq p<\infty$ and any normed space in $v\left(l_{p}\right)$ [3, Theorem 4.1, p. 217] is a $Q_{p}$ space for $2 \leqq p<\infty$. It is also fairly easy to show that for $1<q \leqq 2$, the dual of any normed space in $v\left(l_{a}\right)$ is a $Q_{p}$ space, where $p^{-1}+q^{-1}=1$.

The following lemma singles out a result needed for the theorem; it also shows how Clarkson's "parallelogram" laws figure into the main result.

Lemma. Let $2 \leqq p<\infty, X$ a $Q_{p}$ space, $T: c_{0} \rightarrow X$ a map and $\left(\lambda_{n}\right)$ a sequence such that $\left(\lambda_{n}^{-1}\right) \notin l_{p}$; then there exists a subsequence $\left(n^{\prime}\right)$ of $(n)$ such that $\left|\lambda_{n^{\prime}}\right|\left\|T e_{n^{\prime}}\right\| \rightarrow 0$ as $n^{\prime} \rightarrow \infty$.

Proof. Suppose not; then there exists an $\varepsilon>0$ and an integer $M$, such that $n \geqq M$ implies $\left|\lambda_{n}\right|\left\|T e_{n}\right\| \geqq \varepsilon$. We show by induction that there is some choice of signs so that

$$
\left\|T\left(e_{M} \pm \cdots \pm e_{M+k}\right)\right\|^{p} \geqq \varepsilon^{p} \sum_{j=0}^{k}\left|\hat{\lambda}_{M+j}^{-1}\right|^{p} .
$$

By assumption (2) is true for $k=0$. Suppose that (2) is true for $k=n$ with choice of signs $x_{n}=e_{M} \pm e_{M+1} \pm \cdots \pm e_{M+n}$. Now by (1) and the induction hypothesis we have

$$
\begin{aligned}
&\left\|T\left(x_{n}+e_{M+n+1}\right)\right\|^{D}+\left\|T\left(x_{n}-e_{M+n+1}\right)\right\|^{p} \\
& \geqq 2\left(\left\|T x_{n}\right\|^{p}+\left\|T e_{M+n+1}\right\|^{p}\right) \\
& \geqq 2\left(\varepsilon^{p} \sum_{j=0}^{n}\left|\lambda_{M+j}^{-1}\right|^{p}+\varepsilon^{p}\left|\lambda_{M+n+1}^{-1}\right|^{p}\right) ;
\end{aligned}
$$

so (2) is established. But this is impossible since for all $k$,

$$
\left\|e_{M} \pm e_{M+1} \pm \cdots \pm e_{M+k}\right\|=1, \quad \sum\left|\lambda_{M+j}\right|^{p}
$$

diverges and thus (2) implies that $T$ is unbounded.

THEOREM. A necessary condition for the map $S$ from $c_{0}$ or $l_{\infty}$ into a normed space $Y$ to be factorable through a $Q_{p}$ space, $2 \leqq p<\infty$, is that the sequence $\left(\left\|S e_{n}\right\|\right)$ belong to $l_{p}$. 
Proof. The theorem for $c_{0}$ clearly implies the theorem for $l_{\infty}$. Let $2 \leqq p<\infty$ and suppose $S: c_{0} \rightarrow Y$ can be factored through the $Q_{p}$ space $X$ by the maps $T: c_{0} \rightarrow X$ and $J: X \rightarrow Y$, but that $\left(\left\|S e_{n}\right\|\right) \notin l_{p}$. Let $V: c_{0} \rightarrow c_{0}$ be the map with $V e_{n}=e_{n(n)}$ where the one-one function $\pi$ from $N$ into $N$ ( $N$ the positive integers) is such that, for all $n,\left\|S V e_{n}\right\| \neq 0$ and $\left(\left\|S V e_{n}\right\|\right) \notin$ $l_{p}$. By assumption, $S V$ can be factored through $X$ by the maps $T V$ and $J$, that is, $S V=J T V$. The lemma implies there exists a subsequence $\left(n^{\prime}\right)$ of $(n)$ such that $\left\|S V e_{n^{\prime}}\right\|^{-1}\left\|T V e_{n^{\prime}}\right\| \rightarrow 0$ as $n^{\prime} \rightarrow \infty$. But by continuity of $J$ we have as $n^{\prime} \rightarrow \infty$ :

$$
1=\left\|S V e_{n^{\prime}}\right\|^{-1}\left\|S V e_{n^{\prime}}\right\|=\left\|J\left(\left\|S V e_{n^{\prime}}\right\|^{-1} T V e_{n^{\prime}}\right)\right\| \rightarrow 0 .
$$

This contradiction completes the proof.

A diagonal map on a space $\Lambda$, of sequences, is a map $T_{\lambda}: \Lambda \rightarrow \Lambda$, where $\lambda$ is a sequence $\left(\lambda_{n}\right)$ and $T_{\lambda}\left(\mu_{n}\right)=\left(\lambda_{n} \mu_{n}\right)$.

COROLlaRY. A necessary condition for the diagonal map $T_{\lambda}: l_{1} \rightarrow l_{1}$ to be factorable through a space $X$, whose dual is a $Q_{p}$ space $(2 \leqq p<\infty)$ is that $\lambda=\left(\lambda_{n}\right) \in l_{p}$.

PROOF. If $T_{\lambda}: l_{1} \rightarrow l_{1}$ can be factored through $X$, then $T_{\lambda}^{*}: l_{\infty} \rightarrow l_{\infty}$ can be factored through $X^{*}$. The proof now follows from the theorem and the fact that the adjoint of $T_{\lambda}, T_{\lambda}^{*}$ is the diagonal map $T_{\lambda}: l_{\infty} \rightarrow l_{\infty}$.

In [2] and [3] the following questions were raised:

(i) Is $\bigcap v(B), B \in \mathscr{B}$ (where $\mathscr{B}=$ all infinite dimensional Banach spaces\}), equal to $S$, the variety of all Schwartz spaces; $N$, the variety of all nuclear spaces, or neither?

(ii) Does $v\left(l_{p}\right), 1<p<\infty$, contain $S$ ?

The following examples show that for no $p, 1<p<\infty$, is (ii) true and thus $\bigcap \nu(B), B \in \mathscr{B}$, is properly contained in $S$ [2]. Furthermore we show $\bigcap v\left(l_{p}\right), 1 \leqq p \leqq \infty$, properly contains $N$.

EXAMPLES. Let $\lambda=\left(\lambda_{n}\right)$ be any sequence converging to zero such that, for $k \geqq 1, \sum\left|\lambda_{n}\right|^{k}=\infty$, and let $T_{\lambda}$ be the diagonal map. Let $\Lambda_{0}$ (respectively, $\Lambda_{1}, \Lambda_{2}, \Lambda_{\infty}$ ) be the projective limit of the sequence:

$$
\cdots \stackrel{T_{\lambda}}{\longrightarrow} E \stackrel{T_{\lambda}}{\longrightarrow} E \stackrel{T_{\lambda}}{\longrightarrow} E,
$$

where $E=c_{0}$ (respectively, $E=l_{1}, E=l_{2}, E=l_{\infty}$ ). Each of $\Lambda_{i}, i=0,1,2, \infty$ is a Schwartz-Fréchet space [5, Proposition 9, p. 282], that is not nuclear. $\Lambda_{0}$ and $\Lambda_{\infty}$ (respectively $\Lambda_{1}$ ) do not belong to the variety $v\left(l_{p}\right)$ for $2 \leqq p<\infty$ (respectively $1<p \leqq 2$ ); in particular, for fixed $p$, it is not possible to topologically embed either in a product of $l_{p}$ spaces $2 \leqq p<\infty$ (respectively $1<p \leqq 2$ ). The above follows as the assertions are equivalent to the impossibility of factoring any finite number of iterates of $T_{\lambda}$ through spaces $X$ 
covered by the theorem or the corollary (see the discussion preceding the lemma).

The space $\Lambda_{2}$ is an example of Pietsch [8, Satz 8, p. 122], where he shows that $\Lambda_{2}$ is a subspace of a product of $l_{p}$ spaces for any $p, 1<p<\infty$. Hence $\Lambda_{2} \in v\left(l_{p}\right)$ for all $p, 1 \leqq p \leqq \infty$ (the cases $p=1$ and $p=\infty$ are true for any Schwartz space [3]).

\section{REFERENCES}

1. J. A. Clarkson, Uniformly convex spaces, Trans. Amer. Math. Soc. 40 (1936), 396-414.

2. J. Diestel and S. A. Morris, Remarks on varieties of locally convex linear topological spaces (to appear).

3. J. Diestel, S. A. Morris and S. A. Saxon, Varieties of linear topological spaces, Trans. Amer. Math. Soc. 172 (1972), 207-230.

4. A. Grothendieck, Produits tensoriels topologiques et espaces nucléaires, Mem. Amer. Math. Soc. No. 16, (1955). MR 17, 763.

5. J. Hovarth, Topological vector spaces and distributions. Vol. 1, Addison-Wesley, Reading, Mass., 1966. MR 34 \#4863.

6. J. Lindenstrauss and A. Pelczyński, Absolutely summing operators in $\mathscr{L}_{p}$-spaces and their applications, Studia Math. 29 (1968), 275-326. MR 37 \#6743.

7. J. Lindenstrauss and H. P. Rosenthal, The $\mathscr{L}_{p}$-spaces, Israel J. Math. 7 (1969), 325-349. MR 42 \#5012.

8. A. Pietsch, $l_{p}$-faktorisierbare Operatoren in Banachräumen, Acta Sci. Math. (Szeged) 31 (1970), 117-123. MR 42 \#889.

9. S. A. Saxon, Embedding nuclear spaces in products of an arbitrary Banach space, Proc. Amer. Math. Soc. 34 (1972), 138-140.

Department of Mathematics, Claremont Graduate School, Claremont, CALIFORNIA 91711 